\title{
Comparative pharmacokinetics, safety, and tolerability of two sources of ch14.18 in pediatric patients with high-risk neuroblastoma following myeloablative therapy
}

\author{
Araz Marachelian ${ }^{1} \cdot$ Ami Desai $^{2} \cdot$ Frank Balis $^{2} \cdot$ Howard Katzenstein ${ }^{3}$. \\ Muna Qayed $^{4} \cdot$ Michael Armstrong $^{5}$ Kathleen A. Neville ${ }^{6} \cdot$ Susan L. Cohn $^{7}$ • \\ Mark Bush $^{8} \cdot$ Rudy Gunawan $^{9}$ Allison Pecha Lim ${ }^{10} \cdot$ Malcolm A. Smith ${ }^{11}$. \\ L. Mary Smith ${ }^{10}$
}

Received: 25 November 2015 / Accepted: 26 December 2015 / Published online: 20 January 2016 (C) The Author(s) 2016. This article is published with open access at Springerlink.com

\begin{abstract}
Purpose Dinutuximab (Unituxin ${ }^{\mathrm{TM}}$; ch14.18), a monoclonal antibody against disialoganglioside, improved survival as part of post-consolidation therapy for high-risk neuroblastoma. United Therapeutics Corporation (UTC) assumed ch14.18 production from the National Cancer Institute (NCI); this study evaluates pharmacokinetic comparability, safety, and tolerability of UTC and NCI products.

Methods In this randomized, two-sequence crossover study, 28 patients aged $\leq 8$ years with high-risk neuroblastoma received equivalent ch14.18-UTC or ch14.18-NCI doses. Despite comparable protein content, nominal doses
\end{abstract}

Mark Bush and Rudy Gunawan: Employed by Nuventra Pharma Sciences at the time of writing the manuscript.

Electronic supplementary material The online version of this article (doi:10.1007/s00280-015-2955-9) contains supplementary material, which is available to authorized users.

Araz Marachelian

amarachelian@chla.usc.edu

1 Children's Hospital Los Angeles, University of Southern California, 4650 Sunset Blvd, Los Angeles, CA 90027, USA

2 Children's Hospital of Philadelphia, 3401 Civic Center Blvd, Philadelphia, PA 19104, USA

3 Vanderbilt University School of Medicine, 2200 Pierce Ave 397 PRB, Nashville, TN 37232-6310, USA

4 Aflac Cancer and Blood Disorders Center, Children's Healthcare of Atlanta and Emory University School of Medicine, 1405 Clifton Rd NE, Atlanta, GA 30322, USA

5 Duke University, DUMC 102382, Durham, NC 27710, USA

6 Arkansas Children's Hospital/University of Arkansas for Medical Sciences, 1 Children's Way; Slot 512-23, Little Rock, AR 72202, USA differed: $17.5 \mathrm{mg} / \mathrm{m}^{2} /$ day (ch14.18-UTC) and $25 \mathrm{mg} / \mathrm{m}^{2} /$ day (ch14.18-NCI). Patients received one product during therapy cycles 1 and 2, the other during cycles 3-5. Ch14.18 pharmacokinetic profile characterization used population modeling $\left(\mathrm{NONMEM}^{\circledR}\right.$ version 7.2). A twocompartment model with first-order distribution and elimination processes described pharmacokinetic data. Estimated product parameters were normalized to UTC nominal dose. For pharmacokinetic comparability, the final model was used to estimate exposure ratios (UTC/NCI) and associated $90 \%$ confidence intervals (CIs) for area under the curve from time zero to infinity $\left(\mathrm{AUC}_{\mathrm{inf}}\right)$ and maximum concentration $\left(C_{\max }\right)$. All comparisons were based on a standardized single-dose regimen $\left(17.5 \mathrm{mg} / \mathrm{m}^{2}\right.$ over $\left.10 \mathrm{~h}\right)$.

Results Final-model pharmacokinetic parameters were similar to previously published ch14.18-NCI parameters and comparable for UTC and NCI products. Products' systemic exposures were comparable, with $90 \%$ CIs around

7 Department of Pediatrics, The University of Chicago, $900 \mathrm{E}$ 57th Street, KCBD, Rm 5100, Chicago, IL 60637, USA

8 School of Pharmacy, Wingate University, 515 N. Main Street, Wingate, NC 28174, USA

9 Ionis Pharmaceuticals, 2855 Gazelle Court, Carlsbad, CA 92010, USA

10 United Therapeutics Corporation, 55 TW Alexander Drive, Research Triangle Park, NC 27709, USA

11 National Cancer Institute, 9609 Medical Center Dr, MSC 9739, Bethesda, MD 20892-9739, USA 
ratios for $\mathrm{AUC}_{\mathrm{inf}}(0.96 ; 90 \% \mathrm{CI} 0.88-1.04)$ and $C_{\max }(1.04$; $90 \%$ CI 0.98-1.11) within standard bioequivalence bounds (90 \% CI 0.80-1.25). Products' adverse events were similar and consistent with those previously reported.

Conclusions Equivalent actual ch14.18-UTC and ch14.18-NCI doses produced comparable exposures, with no notable safety or tolerability differences.

Keywords ch14.18 - Dinutuximab - Pharmacokinetics · Safety $\cdot$ Tolerability $\cdot$ Unituxin

\section{Introduction}

Neuroblastoma, which is a tumor of the autonomic nervous system, accounts for approximately $7 \%$ of cancers in children $<15$ years of age [1]; $90 \%$ of patients are $<5$ years of age at diagnosis [1]. The disease is heterogeneous, complex, and frequently aggressive [2]. At diagnosis, approximately $40 \%$ of patients have high-risk disease [3], based on factors such as age, disease stage, and biologic markers (e.g., unfavorable histopathology, tumor amplification of $M Y C N$ oncogene) [4]. High-risk neuroblastoma is treated with dose-intensive chemotherapy and surgery, followed by myeloablative chemotherapy with autologous stem cell transplantation (ASCT), local radiation therapy, and maintenance with isotretinoin [5, 6]. Despite this intensive treatment, many patients relapse or have treatment-refractory disease, and 5-year event-free survival rates are $\leq 50 \%[4,7]$.

Disialoganglioside $\left(\mathrm{G}_{\mathrm{D} 2}\right)$ is a surface glycolipid antigen that is strongly expressed on neuroblastoma tumor cells, with limited expression in normal human tissues [8]. $G_{D 2}$ is an important molecular target for immunotherapeutic approaches to treating neuroblastoma, and anti- $\mathrm{G}_{\mathrm{D} 2}$ monoclonal antibodies are efficacious in patients with high-risk neuroblastoma.

Dinutuximab (Unituxin ${ }^{\mathrm{TM}}$ ), formerly called ch14.18, is a murine-human chimeric anti- $\mathrm{G}_{\mathrm{D} 2}$ monoclonal antibody [9]. Initial trials demonstrated that ch14.18 at a dose of $25 \mathrm{mg} / \mathrm{m}^{2}$ infused over $10 \mathrm{~h}$ daily for 4 consecutive days could be incorporated into treatment regimens containing isotretinoin and the immunomodulators sargramostim and aldesleukin [10-12]. Subsequently, the Children's Oncology Group (COG) conducted a randomized phase 3 clinical trial (ANBL0032) comparing ch14.18 administered with isotretinoin, sargramostim, and aldesleukin versus isotretinoin alone in patients with high-risk neuroblastoma who had responded to induction therapy, surgery, ASCT, and radiotherapy [12]. The trial demonstrated improved eventfree survival $(p=0.01)$ and overall survival $(p=0.02)$ at 2 years on the immunotherapy arm [12]. Based on the results of this trial, dinutuximab received US Food and Drug Administration (FDA), and European Medicines
Agency (EMA) approval for the treatment of high-risk neuroblastoma.

As part of a collaborative research agreement and development agreement (CRADA) with the National Cancer Institute (NCI), United Therapeutics Corporation (UTC) has licensed ch14.18 and assumed production. The nominal (i.e., labeled) dose of the UTC product $\left(17.5 \mathrm{mg} / \mathrm{m}^{2}\right)$ differs from the dose of the prior NCI product $\left(25 \mathrm{mg} / \mathrm{m}^{2}\right)$ because of a difference in the extinction coefficient used to determine the protein concentration during the manufacturing process. Despite the change in nominal dosing, the amount of antibody delivered per dose is equivalent for the two products. Corrections for this difference in the products' nominal dose must be made when comparing dosedependent pharmacokinetic parameters, such as clearance and volumes of distribution.

The primary objective of this study was to compare the pharmacokinetics of ch14.18 manufactured by these two independent facilities (i.e., NCI and UTC). The secondary objective was to compare the products' safety and tolerability profiles.

\section{Materials and methods}

\section{Study design}

Study DIV-NB-201 was a phase 2 randomized, open-label, two-sequence crossover trial evaluating ch14.18 in patients with high-risk neuroblastoma scheduled to receive immunotherapy. The clinical trial was conducted in accordance with the ethical principles of the Declaration of Helsinki and the International Conference on Harmonization E6 Good Clinical Practice Guideline. The protocol was approved by the Institutional Review Board at each participating site, and the parents or guardians provided written informed consent with patient assent, as appropriate. Patients were randomized 1:1 to receive either ch14.18UTC or ch14.18-NCI during cycles 1 and 2, followed by ch14.18 from the other product during cycles 3-5. Eligible patients were randomized between 56 and 105 days after ASCT. Randomization must have occurred after the completion of tumor assessments post-ASCT and radiotherapy, if applicable.

\section{Patients and treatment}

Eligible patients were $\leq 8$ years of age, had a diagnosis of high-risk neuroblastoma, and had completed standard induction therapy, surgery, myeloablative therapy and ASCT, and local radiotherapy to the primary tumor if indicated. Patients must have achieved a partial response or better per International Neuroblastoma Response Criteria 
(INRC) [13] at the primary site, soft tissue metastases, bone metastases, and bone marrow response at the preASCT evaluation. Prior to enrollment, a determination of residual disease was performed, and patients could not have progressive disease per INRC except for protocolspecified bone marrow response to account for sampling errors. Patients were also required to have a Lansky performance status of $\geq 50 \%$; a total absolute phagocyte count $\geq 1000 / \mu \mathrm{L}$; adequate renal, hepatic, cardiac, pulmonary, and central nervous system function; and a life expectancy of $\geq 2$ months. Patients were excluded if they had received prior anti- $\mathrm{G}_{\mathrm{D} 2}$ antibody therapy or had prior vaccine therapy for the treatment of neuroblastoma. Patients were also excluded if they had received or planned to receive anticancer therapies, cytokines, or growth factors not included in the prescribed protocol therapy during the study or immunosuppressive drugs other than for acute allergic reactions and anaphylaxis during the study.

The study material manufactured by NCI used a theoretical extinction coefficient of 1.00 to calculate the concentration of antibody, whereas UTC material used an actual extinction coefficient of 1.41 to determine the antibody concentration. Thus, each $25 \mathrm{mg} / \mathrm{m}^{2}$ dose of ch14.18-NCI contains the same amount of $\mathrm{ch} 14.18$ as a $17.5-\mathrm{mg} / \mathrm{m}^{2}$ dose of ch14.18-UTC, making the respective dosing equivalent despite differences in the nominal doses. The dosing schema in this study is summarized in Table 1. During the first five cycles, patients received ch14.18-UTC or ch14.18NCI intravenously (IV) over 10-20 h daily for four consecutive days repeated every 28 days, with one product administered during cycles 1 and 2 and the other product during cycles 3, 4, and 5. All patients received sargramostim IV or subcutaneously (SC) $\left(250 \mathrm{mcg} / \mathrm{m}^{2} /\right.$ day for 14 days $)$ on cycles 1,3 , and 5 prior to, during, and following ch14.18, and aldesleukin IV ( $3 \mathrm{MIU} / \mathrm{m}^{2} /$ day for 4 days as a continuous infusion for the first week, followed by $4.5 \mathrm{MIU} / \mathrm{m}^{2}$ / day for 4 days as a continuous infusion concurrently with ch14.18 for the second week) during cycles 2 and 4. In addition, all patients received six cycles of isotretinoin over 14 days $\left(80 \mathrm{mg} / \mathrm{m}^{2} /\right.$ day orally twice daily for $>12 \mathrm{~kg}$ and $2.67 \mathrm{mg} / \mathrm{kg}$ orally twice daily for $\leq 12 \mathrm{~kg}$ ) after completion of ch14.18 therapy.

In addition to study treatment, supportive care measures, including the use of narcotics and other concomitant medications, were required for the treatment of anticipated toxicities.

\section{Assessments}

Pharmacokinetic blood samples for the determination of ch14.18 plasma concentrations were obtained at 22 time points over the course of the treatment (Table 1). Samples were drawn prior to the first dose of sargramostim on cycle
1 and the first ch14.18 infusion on cycle 3; after the end of each daily ch14.18 infusion; and 10-14 h, 3-5 days, and 8-11 days after the fourth ch14.18 infusion on cycles 1 and 3. Sampling during cycles 2 and 4 occurred prior to aldesleukin, prior to the first ch14.18 dose, and at the end of the fourth daily ch14.18 infusion on cycle 4 only. A final sample was obtained at study end, within 2 weeks of the final isotretinoin dose on cycle 6. Blood samples for the analysis of human anti-chimeric antibody (HACA) were obtained prior to ch14.18 dosing in each cycle. Plasma from each sample was isolated by centrifugation, frozen, and shipped to Burleson Research Technologies, Morrisville, NC, for storage. Samples were shipped to BioAgilytix, Durham, NC, where ch14.18 and HACA plasma concentrations were measured using validated assays: a sandwich immunoassay employing an electrochemiluminescence platform to measure ch14.18, and a Meso Scale Discovery electrochemiluminescent assay (Meso Scale Diagnostics, Rockville, MD) with a lower limit of quantification of a titer of 10 to measure HACA.

Because of the study complexity and data limitations, a model-based approach, rather than a traditional noncompartmental bioequivalence analysis, was used to assess the pharmacokinetic comparability of ch14.18-UTC and ch14.18-NCI. Detailed pharmacokinetic data from nine patients with high-risk neuroblastoma enrolled in a previous pharmacokinetic study of ch14.18-NCI at the Children's Hospital of Philadelphia by Desai [14] were used to develop a structural population pharmacokinetic model. The final model was a two-compartment model with firstorder distribution and elimination processes. To account for the effects of body size on pharmacokinetic parameters, actual body weight was included as a predetermined allometric covariate on all clearance and volume of distribution parameters. Estimated pharmacokinetic parameters included clearance from the central compartment (CL), distributional clearance $(Q)$, volume of the central compartment (V1), volume of the peripheral compartment (V2), steady-state volume of distribution $\left(V_{\mathrm{ss}}\right)$, first-order elimination rate constant (Kel), and first-order distribution rate constants (central-to-peripheral [Kcp]; peripheral-tocentral $[\mathrm{Kpc}])$. The final structural model for the data from the prior Desai study was then used to estimate pharmacokinetic parameters for ch14.18-UTC and ch14.18-NCI in the formal comparability study (DIV-NB-201). This staged analysis approach was taken to avoid the potential for inflated alpha error associated with iterative model development using the formal comparability data from DIV-NB-201.

Because calculations of some pharmacokinetic parameters are dependent on the nominal dose of drug, differences in the nominal doses of ch14.18-UTC and ch14.18NCI will affect pharmacokinetic parameter estimates. In 
the Desai study [14], pharmacokinetic parameter estimates were based on nominal dosing units of ch14.18-NCI. Therefore, when comparing dose-dependent pharmacokinetic parameters generated using UTC and NCI nominal doses, appropriate corrections were made.

To formally assess the pharmacokinetic bioequivalence of the UTC and NCI products, maximum concentration $\left(C_{\max }\right)$ and area under the curve from time zero to infinity $\left(\mathrm{AUC}_{\text {inf }}\right)$ were calculated using the population pharmacokinetic parameter estimates of CL and V from DIVNB-201 and appropriate closed-form equations for $C_{\max }$ and $\mathrm{AUC}_{\text {inf }}$. Variability in exposure estimates was captured by sampling the posterior distribution of pharmacokinetic parameter estimates using the NONMEM ${ }^{\circledR}$ Markov Chain Monte Carlo methodology. To allow for valid comparisons, all calculations were based on a standardized single dose of $17.5 \mathrm{mg} / \mathrm{m}^{2}$ infused over $10 \mathrm{~h}$, using nominal UTC doses. Pharmacokinetic bioequivalence was assessed by calculation of ratios (UTC/NCI) for $\mathrm{AUC}_{\text {inf }}$ and $C_{\max }$ with $90 \%$ confidence interval (CI) of the ratios. $\mathrm{AUC}_{\mathrm{inf}}$ was the primary comparability end point. Bioequivalence was established if the $90 \%$ CIs for the exposure ratios were completely contained within accepted bioequivalence bounds (0.80-1.25).

Safety assessments included adverse event reporting, physical examinations, clinical laboratory assessments, and treatment-related changes in electrocardiograms (ECGs). Safety analyses were performed on all patients receiving at least one study drug dose. No inferential statistical analyses of safety data were planned.

\section{Results}

Twenty-eight patients were enrolled, and 14 patients were randomized to each treatment sequence. Patient characteristics are summarized in Table 2 with no differences in demographics by sequence. A summary of concomitant medications used by patients during cycles $1-5$ is presented in Supplemental Table 1. Patients' completion or discontinuation of study therapy is summarized in Table 3. One patient was excluded from the pharmacokinetic analysis because of a neutralizing antibody response that interfered with measurement of ch14.18 concentration. All 28 patients received at least one dose of ch14.18 and were included in the safety analysis. Seven patients discontinued the study prior to completion of the planned six cycles; reasons were disease progression $(n=2)$, adverse events $(n=2)$, or withdrawal of consent $(n=1)$. Two patients received cycles 1-5 in the USA and returned to their home country to complete cycle 6 . Ch14.18 was administered over a median duration of $11 \mathrm{~h}$ (range 10-20 h).

\section{Immunogenicity}

Six of 27 patients had detectable HACA during the study. Only one patient $(17 \%)$ had a pharmacokinetic-neutralizing response (detected in cycle 3) and was therefore excluded from the pharmacokinetic analysis.

\section{Pharmacokinetics}

Representative concentration time profiles for ch14.18UTC and ch14.18-NCI from a single patient are presented in Fig. 1a (semilog), b (linear). A comparison of the pharmacokinetic profiles indicates similar exposures for both products. Population mean concentration time profiles are shown in Supplemental Figure 1. Summary statistics of post hoc pharmacokinetic parameters are presented in Table 4 for each product, separately and combined. Dosedependent pharmacokinetic parameters were normalized to the nominal ch14.18-UTC dose. Clearance, volumes of distribution, and rate constants were equivalent for the NCIand UTC-manufactured products.

Following standardized single-dose regimens, population pharmacokinetic estimates for $\mathrm{AUC}_{\text {inf }}$ were $431 \mu \mathrm{g} \cdot \mathrm{h} /$ $\mathrm{mL}$ for ch14.18-UTC and $413 \mu \mathrm{g} \cdot \mathrm{h} / \mathrm{mL}$ for ch14.18-NCI $($ ratio $=1.04 ; 90 \%$ CI 0.98-1.11). Population pharmacokinetic estimates for $C_{\max }$ were $6.57 \mu \mathrm{g} / \mathrm{mL}$ and $6.88 \mu \mathrm{g} / \mathrm{mL}$, respectively (ratio $=0.96 ; 90 \% \mathrm{CI} 0.88-1.04$ ). The $90 \%$ CIs for exposure ratios of $\mathrm{AUC}_{\text {inf }}$ and $C_{\max }$ were contained within the standard bioequivalence bounds (0.80-1.25), consistent with comparable exposure between products.

\section{Safety}

All 28 randomized patients were included in the safety analyses and had at least one treatment-related adverse event (TRAE) overall and at least one TRAE attributed to ch14.18. Overall, a total of 1945 TRAEs were reported, with most being grades $1-3$. The most commonly reported TRAEs included pyrexia (100\%), hypoalbuminemia (96\%), hypokalemia (96\%), hyponatremia (82\%), cough $(75 \%)$, increased alanine aminotransferase (ALT) $(68 \%)$, anemia $(68 \%)$, hypocalcemia (68\%), pain (68\%), pruritus (68\%), increased aspartate aminotransferase (AST) (64\%), hypertriglyceridemia (64\%), and abdominal pain $(61 \%)$. Although differences between products were seen for individual adverse events, evaluation over the entire study showed no notable differences in TRAE incidence by manufacturer, either overall or attributable to ch14.18. Pain-related TRAEs were generally similar between treatment sequences; the most commonly reported pain-related events in the ch14.18-UTC and ch14.18-NCI groups were pain (59 and $44 \%$ ), abdominal pain (48 and $41 \%$ ), and 
Table 1 Dosing schema and pharmacokinetic assessment schedule

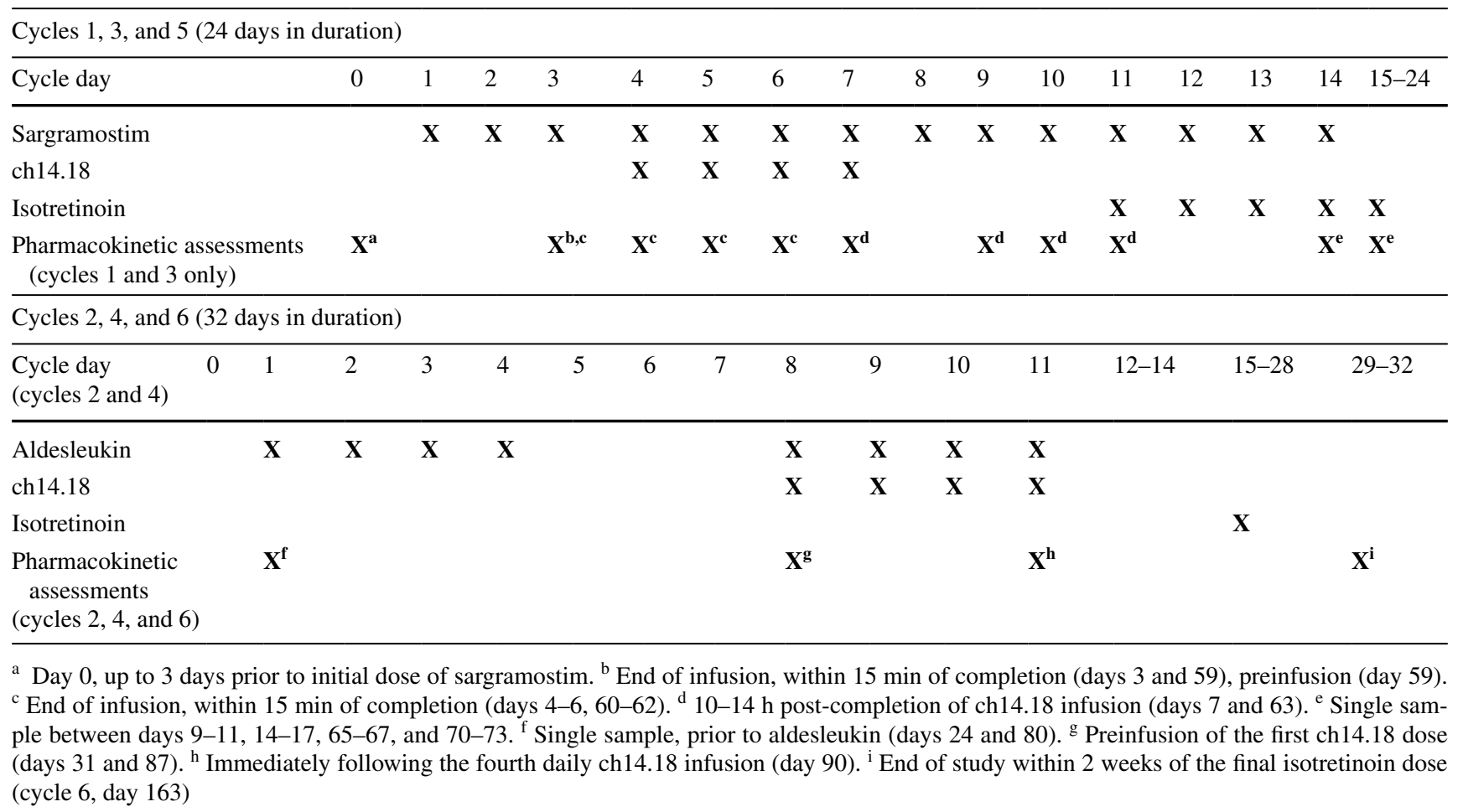

pain in extremity (33 and $41 \%$ ), respectively. Pain-related TRAEs were most commonly reported during cycles 1,2 , and 4 (93, 74, and $71 \%$, respectively). Allergictype adverse events were also generally similar between ch14.18-UTC and ch14.18-NCI groups, with the most commonly reported events (i.e., $\geq 15 \%$ of patients) including urticaria (30 vs. $26 \%$ ), peripheral edema (15 vs. $11 \%$ ), pruritus (63 vs. $52 \%$ ), and rash ( 26 vs. $26 \%$ ), respectively, and occurred most frequently in cycles 1,2 , and 4 .

Table 5 summarizes grade 3 or higher TRAEs considered by the investigator to be attributable to ch14.18 in $\geq 10 \%$ of patients. The most common grade $\geq 3$ events were pyrexia, anemia, hypokalemia, and hyponatremia, with no discernible differences between study drugs. Other safety assessments, including clinical laboratories, physical examinations, and ECGs, were generally consistent between UTC- and NCI-manufactured ch14.18.

\section{Discussion}

The NCI-manufactured ch14.18 was used in the pivotal randomized phase 3 trial that demonstrated the efficacy of ch14.18 combined with sargramostim, aldesleukin, and isotretinoin administered as continuation therapy for highrisk neuroblastoma [12]. This randomized crossover study comparing equivalent doses of ch14.18-UTC $\left(17.5 \mathrm{mg} / \mathrm{m}^{2}\right)$ and ch14.18-NCI $\left(25 \mathrm{mg} / \mathrm{m}^{2}\right)$ was conducted to confirm equivalence of the pharmacokinetic and safety profiles of the two products.

Pharmacokinetic parameters were estimated by fitting a two-compartment pharmacokinetic model to individual concentration-time data. The products were formally compared using the results of a model-based bioequivalence analysis, which showed equivalent systemic exposures as measured by the $\mathrm{AUC}_{\mathrm{inf}}$ for ch14.18-UTC and ch14.18$\mathrm{NCI}$, and $90 \%$ CIs about the geometric least-squares mean ratios for $\mathrm{AUC}_{\text {inf }}$ and $C_{\max }$ within standard bioequivalence bounds (90\% CI 0.80-1.25). Clearance, volume of distribution, and rate constants were also equivalent between the UTC and NCI products.

For this analysis, dose-dependent pharmacokinetic parameters were derived using the ch14.18-UTC nominal dose of $17.5 \mathrm{mg} / \mathrm{m}^{2}$. Historical parameters, such as CL and volumes of distribution, for the ch14.18-NCI material were derived using a $25-\mathrm{mg} / \mathrm{m}^{2}$ dose and require a correction factor of 0.7 for comparison with ch14.18-UTC material.

Data from an independent study (CHP1002) were used to develop a pharmacokinetic model for ch14.18. This model was then used to estimate pharmacokinetic parameters for the comparability study (DIV-NB-201). This approach was taken to avoid the potential for inflated alpha error associated with iterative model development based on the formal comparability data (DIV-NB-201). A model-based approach (rather than a traditional noncompartmental bioequivalence analysis) was chosen to assess 
Table 2 Patient demographic and baseline characteristics

\begin{tabular}{|c|c|c|}
\hline Characteristic & $\begin{array}{l}\text { Sequence } 1(n=14) \\
\text { (UTC/NCI) }\end{array}$ & $\begin{array}{l}\text { Sequence } 2(n=14) \\
(\text { NCI/UTC) }\end{array}$ \\
\hline $\begin{array}{l}\text { Mean age at randomization (range) } \\
\text { (years) }\end{array}$ & $4(2-7)$ & $4(1-9)$ \\
\hline Male gender, $n(\%)$ & $8(57)$ & $8(57)$ \\
\hline \multicolumn{3}{|l|}{ Ethnicity, $n(\%)$} \\
\hline Hispanic & $4(29)$ & $2(14)$ \\
\hline Not Hispanic & $10(71)$ & $12(86)$ \\
\hline \multicolumn{3}{|l|}{ Race, $n(\%)$} \\
\hline White & $12(86)$ & $11(79)$ \\
\hline Asian & 0 & $1(7)$ \\
\hline Black/African American & $2(14)$ & $1(7)$ \\
\hline Unknown & 0 & $1(7)$ \\
\hline \multicolumn{3}{|l|}{ Pre-ASCT response, $n(\%)$} \\
\hline Complete response & $5(36)$ & $3(21)$ \\
\hline Very good partial response & $5(36)$ & $4(29)$ \\
\hline Partial response & $4(29)$ & $7(50)$ \\
\hline \multicolumn{3}{|l|}{ Number of ASCT, $n(\%)$} \\
\hline Single & $13(93)$ & $14(100)$ \\
\hline Tandem $^{\mathrm{a}}$ & $1(7)$ & 0 \\
\hline Prior chemotherapy, $n(\%)$ & $14(100)$ & $14(100)$ \\
\hline Radiotherapy, $n(\%)$ & $12(86)^{b}$ & $13(93)$ \\
\hline Cancer-related surgery, $n(\%)$ & $12(86)^{\mathrm{c}}$ & $11(79)$ \\
\hline
\end{tabular}

ASCT autologous stem cell transplantation, NCI National Cancer Institute, UTC United Therapeutics Corporation

${ }^{a}$ Patients were required to undergo ASCT (first transplant for tandem transplant patients) within 9 months after starting the first induction chemotherapy for high-risk neuroblastoma. In addition, patients were required to enroll in the study within 105 days post-ASCT (date of second transplant for tandem patients) such that study day 0 (first dose of sargramostim) occurred within 110 days post-transplantation

b Radiotherapy may have been waived for patients who either had a small adrenal mass that was completely resected initially or who never had an identifiable primary tumor

${ }^{c}$ Patients may not have had an identifiable primary tumor
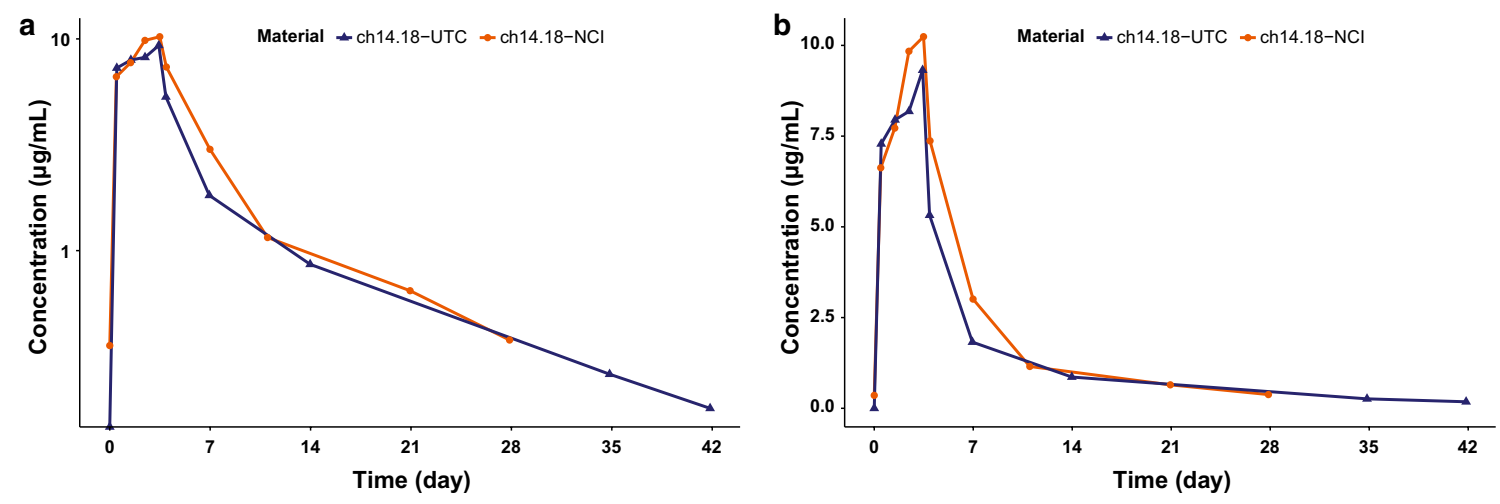

Fig. 1 Representative semilog (a) and linear (b) concentration-time profiles from a single patient for ch14.18-UTC and ch14.18-NCI

the pharmacokinetic comparability of ch14.18-UTC and ch14.18-NCI because of data limitations and the complexity of DIV-NB-201. The pharmacokinetic parameters derived from the population model for the CHP1002 study were comparable to those previously published by Desai et al. [14]. The Desai analysis was also based on a twocompartment structural model; however, the methodology used for parameter estimation differed from the current 
Table 3 Patients' completion or discontinuation of study therapy

\begin{tabular}{|c|c|c|}
\hline Disposition & $\begin{array}{l}\text { Sequence } 1 \\
\text { (UTC/NCI), } \\
n(\%)\end{array}$ & $\begin{array}{l}\text { Sequence } 2 \\
(\mathrm{NCI} / \mathrm{UTC}), \\
n(\%)\end{array}$ \\
\hline Safety population, $n$ & 14 & 14 \\
\hline Pharmacokinetic population, $n$ & $13^{\mathrm{a}}$ & 14 \\
\hline Completed all study therapy & $9(64)$ & $12(86)$ \\
\hline \multicolumn{3}{|l|}{ Discontinued study therapy } \\
\hline Cycle 1 & $1(7)$ & 0 \\
\hline Cycle 2 & 0 & $1(7)$ \\
\hline Cycle 3 & $2(14)$ & 0 \\
\hline Cycle 4 & 0 & $1(7)$ \\
\hline Cycle 5 & $2(14)^{b}$ & 0 \\
\hline \multicolumn{3}{|l|}{ Reason for study discontinuation } \\
\hline Disease progression & $1(7)$ & $1(7)$ \\
\hline Adverse event & $1(7)^{c}$ & $1(7)^{d}$ \\
\hline Consent withdrawn & $1(7)$ & 0 \\
\hline Moved out of country & $2(14)^{b}$ & 0 \\
\hline
\end{tabular}

NCI National Cancer Institute, UTC United Therapeutics Corporation

a One patient excluded because of interfering human anti-chimeric antibodies, for the pharmacokinetic assay

b Patients completed cycles 1-5 and went on to complete scheduled course of isotretinoin in their country

c Patient discontinued during cycle 3 due to serum sickness

${ }^{\mathrm{d}}$ Patient discontinued during cycle 2 due to neuropathy

analysis, which utilized a population pharmacokinetic approach. In addition, Desai et al. used different pharmacokinetic software (MLAB; Civilized Software, Silver Spring, MD). Despite these differences, mean pharmacokinetic parameters for the CHP1002 study were comparable between the Desai analysis and the population model. When pharmacokinetic parameters are expressed in terms of nominal ch14.18-NCI dosing units, estimates for the Desai analysis and the population pharmacokinetic analysis, respectively, are similar (CL, 2.1 vs. $1.9 \mathrm{~L} / \mathrm{d} / \mathrm{m}^{2} ; \mathrm{Vl}, 2.2$ vs. $1.9 \mathrm{~L}$; Kel, 0.026 vs. $0.027 \mathrm{l} / \mathrm{h}$; Kcp, 0.021 vs. 0.023 $1 / \mathrm{h}$; and Kpc, 0.010 vs. 0.015 1/h).

Overall, the TRAEs (including allergic-type events) observed in this study were consistent with those reported in other studies in which ch14.18 was administered with growth factors and cytokines $[12,15]$. The most commonly reported adverse events in $>60 \%$ of patients, regardless of manufacturer, were pyrexia, hypoalbuminemia, hypokalemia, hyponatremia, cough, increased ALT, anemia, hypocalcemia, pain, pruritus, increased AST, hypertriglyceridemia, and abdominal pain.

The ANBL0032 study demonstrated that approximately $17 \%$ of patients treated with ch14.18 develop HACA, with $<5 \%$ having a neutralizing antibody response in a biological assay (data on file, United Therapeutics Corporation).
Table 4 Summary of individual post hoc pharmacokinetic parameter estimates for ch14.18

\begin{tabular}{lccl}
\hline $\begin{array}{l}\text { Parameter, mean } \\
(\mathrm{SD})\end{array}$ & $\begin{array}{l}\text { ch14.18-UTC } \\
(n=26)\end{array}$ & $\begin{array}{l}\text { ch14.18-NCI } \\
(n=25)\end{array}$ & $\begin{array}{l}\text { ch14.18-UTC } \\
\text { and ch14.18-NCI } \\
\text { combined } \\
(n=27)\end{array}$ \\
\hline $\mathrm{CL}(\mathrm{L} / \mathrm{d})$ & $0.683(0.307)$ & $0.75(0.32)$ & $0.709(0.315)$ \\
$\mathrm{CL}\left(\mathrm{L} / \mathrm{d} / \mathrm{m}^{2}\right)$ & $1.09(0.457)$ & $1.17(0.455)$ & $1.12(0.457)$ \\
$Q(\mathrm{~L} / \mathrm{d})$ & $0.767(0.163)$ & $0.956(0.213)$ & $0.857(0.198)$ \\
$Q\left(\mathrm{~L} / \mathrm{d} / \mathrm{m}^{2}\right)$ & $1.20(0.026)$ & $1.46(0.023)$ & $1.32(0.056)$ \\
$\mathrm{V} 1(\mathrm{~L})$ & $1.43(0.403)$ & $1.36(0.42)$ & $1.40(0.404)$ \\
$\mathrm{V} 1\left(\mathrm{~L} / \mathrm{m}^{2}\right)$ & $2.23(0.29)$ & $2.05(0.28)$ & $2.15(0.28)$ \\
$\mathrm{V} 2(\mathrm{~L})$ & $3.94(1.1)$ & $3.76(1.09)$ & $3.85(1.07)$ \\
$\mathrm{V} 2\left(\mathrm{~L} / \mathrm{m}^{2}\right)$ & $6.10(0.42)$ & $5.65(0.40)$ & $5.87(0.39)$ \\
$V_{\mathrm{ss}}(\mathrm{L})$ & $5.38(1.47)$ & $5.12(1.49)$ & $5.25(1.45)$ \\
$V_{\mathrm{ss}}\left(\mathrm{L} / \mathrm{m}^{2}\right)$ & $8.32(0.57)$ & $7.70(0.57)$ & $8.03(0.54)$ \\
$\mathrm{Kel}(1 / \mathrm{d})$ & $0.489(0.211)$ & $0.569(0.232)$ & $0.520(0.221)$ \\
$\mathrm{Kcp}(1 / \mathrm{d})$ & $0.552(0.073)$ & $0.723(0.103)$ & $0.630(0.090)$ \\
$\mathrm{Kpc}(1 / \mathrm{d})$ & $0.199(0.018)$ & $0.259(0.021)$ & $0.228(0.018)$ \\
\hline
\end{tabular}

$C L$ clearance from the central compartment, $K c p$ first-order distribution rate constant (central-to-peripheral), $\mathrm{Kel}$ first-order elimination rate constant, $K p c$ first-order distribution rate constant (peripheralto-central), NCI National Cancer Institute, $Q$ distributional clearance, UTC United Therapeutics Corporation, VI volume of the central compartment, $V 2$ volume of the peripheral compartment, $V_{\mathrm{ss}}$ steadystate volume of distribution

Table 5 Grade 3 or higher treatment-related adverse events ( $\geq 10 \%$ )

\begin{tabular}{lll}
\hline Adverse events, $n(\%)$ & $\begin{array}{l}\text { ch14.18-UTC } \\
(n=27)\end{array}$ & $\begin{array}{l}\text { ch14.18-NCI } \\
(n=27)\end{array}$ \\
\hline$\geq 1$ Adverse event & $22(81.5)$ & $23(85.2)$ \\
Pyrexia & $13(48.1)$ & $12(44.4)$ \\
Anemia & $6(22.2)$ & $9(33.3)$ \\
Hypokalemia & $7(25.9)$ & $7(25.9)$ \\
Hyponatremia & $5(18.5)$ & $5(18.5)$ \\
Platelet count decreased & $4(14.8)$ & $5(18.5)$ \\
ALT increased & $4(14.8)$ & $1(3.7)$ \\
Lymphocyte count decreased & $2(7.4)$ & $3(11.1)$ \\
Pain-related adverse events & & \\
Pain & $5(18.5)$ & $2(7.4)$ \\
Pain in extremity & $2(7.4)$ & $3(11.1)$ \\
Abdominal pain & $2(7.4)$ & $3(11.1)$ \\
Hypocalcemia & $3(11.1)$ & $2(7.4)$ \\
Hypotension & $2(7.4)$ & $3(11.1)$ \\
Neutrophil count decreased & $2(7.4)$ & $3(11.1)$ \\
Hypoxia & $1(3.7)$ & $3(11.1)$ \\
Urine output decreased & $3(11.1)$ & $1(3.7)$ \\
\hline
\end{tabular}

$A L T$ alanine aminotransferase, NCI National Cancer Institute, UTC United Therapeutics Corporation

a ALT increases were transient 
In this study, 6/27 (22\%) of patients had confirmed HACA, and only one patient was excluded from the pharmacokinetic analysis due to a neutralizing antibody response.

In summary, the current analysis confirms that the ch14.18-UTC dose of $17.5 \mathrm{mg} / \mathrm{m}^{2}$ is comparable to the ch14.18-NCI dose of $25 \mathrm{mg} / \mathrm{m}^{2}$ in terms of systemic exposure and with no notable safety and tolerability differences.

Acknowledgments Writing assistance was provided by Julia D'Ambrosio, $\mathrm{PhD}$, of SciStrategy Communications. This study was sponsored by United Therapeutics Corporation.

\section{Compliance with ethical standards}

Conflict of interest This study was sponsored by United Therapeutics Corporation. Allison Lim and L. Mary Smith are employees of Untied Therapeutics with stock ownership. Mark Bush received remuneration from United Therapeutics for consulting/advisory services. Susan Cohn reports United Therapeutics stock ownership, and funding for travel to a United Therapeutics sponsored meeting. Frank Balis, Ami Desai, and Kathleen Neville report funding from United Therapeutics. All other authors declare no conflicts of interest.

Open Access This article is distributed under the terms of the Creative Commons Attribution 4.0 International License (http://creativecommons.org/licenses/by/4.0/), which permits unrestricted use, distribution, and reproduction in any medium, provided you give appropriate credit to the original author(s) and the source, provide a link to the Creative Commons license, and indicate if changes were made.

\section{References}

1. Modak S, Cheung NK (2007) Disialoganglioside directed immunotherapy of neuroblastoma. Cancer Investig 25(1):67-77. doi:10.1080/07357900601130763

2. Park JR, Eggert A, Caron H (2010) Neuroblastoma: biology, prognosis, and treatment. Hematol Oncol Clin North Am 24(1):65-86. doi:10.1016/j.hoc.2009.11.011

3. Friedman GK, Castleberry RP (2007) Changing trends of research and treatment in infant neuroblastoma. Pediatr Blood Cancer 49(7 Suppl):1060-1065. doi:10.1002/pbc.21354

4. Matthay KK, George RE, Yu AL (2012) Promising therapeutic targets in neuroblastoma. Clin Cancer Res 18(10):2740-2753. doi:10.1158/1078-0432.ccr-11-1939

5. Maris JM (2010) Recent advances in neuroblastoma. N Engl J Med 362(23):2202-2211. doi:10.1056/NEJMra0804577

6. Modak S, Cheung NK (2010) Neuroblastoma: therapeutic strategies for a clinical enigma. Cancer Treat Rev 36(4):307-317. doi:10.1016/j.ctrv.2010.02.006
7. Cohn SL, Pearson AD, London WB, Monclair T, Ambros PF, Brodeur GM, Faldum A, Hero B, Iehara T, Machin D, Mosseri V, Simon T, Garaventa A, Castel V, Matthay KK (2009) The International Neuroblastoma Risk Group (INRG) classification system: an INRG Task Force report. J Clin Oncol 27(2):289-297. doi:10.1200/jco.2008.16.6785

8. Berois N, Osinaga E (2014) Glycobiology of neuroblastoma: impact on tumor behavior, prognosis, and therapeutic strategies. Front Oncol 4:114. doi:10.3389/fonc.2014.00114

9. Soman G, Kallarakal AT, Michiel D, Yang X, Saptharish N, Jiang H, Giardina S, Gilly J, Mitra G (2012) Analytical characterization of ch14.18: a mouse-human chimeric disialogangliosidespecific therapeutic antibody. MAbs 4(1):84-100. doi:10.4161/ mabs.4.1.18566

10. Ozkaynak MF, Sondel PM, Krailo MD, Gan J, Javorsky B, Reisfeld RA, Matthay KK, Reaman GH, Seeger RC (2000) Phase I study of chimeric human/murine anti-ganglioside G(D2) monoclonal antibody (ch14.18) with granulocyte-macrophage colonystimulating factor in children with neuroblastoma immediately after hematopoietic stem-cell transplantation: a Children's Cancer Group Study. J Clin Oncol 18(24):4077-4085

11. Gilman AL, Ozkaynak MF, Matthay KK, Krailo M, Yu AL, Gan J, Sternberg A, Hank JA, Seeger R, Reaman GH, Sondel PM (2009) Phase I study of ch14.18 with granulocyte-macrophage colony-stimulating factor and interleukin-2 in children with neuroblastoma after autologous bone marrow transplantation or stem-cell rescue: a report from the Children's Oncology Group. J Clin Oncol 27(1):85-91. doi:10.1200/jco.2006.10.3564

12. Yu AL, Gilman AL, Ozkaynak MF, London WB, Kreissman SG, Chen HX, Smith M, Anderson B, Villablanca JG, Matthay KK, Shimada H, Grupp SA, Seeger R, Reynolds CP, Buxton A, Reisfeld RA, Gillies SD, Cohn SL, Maris JM, Sondel PM (2010) Anti-GD2 antibody with GM-CSF, interleukin-2, and isotretinoin for neuroblastoma. N Engl J Med 363(14):1324-1334. doi:10.1056/NEJMoa0911123

13. Brodeur GM, Pritchard J, Berthold F, Carlsen NL, Castel V, Castelberry RP, De Bernardi B, Evans AE, Favrot M, Hedborg F et al (1993) Revisions of the international criteria for neuroblastoma diagnosis, staging, and response to treatment. J Clin Oncol 11(8):1466-1477

14. Desai AV, Fox E, Smith LM, Lim AP, Maris JM, Balis FM (2014) Pharmacokinetics of the chimeric anti-GD2 antibody, ch14.18, in children with high-risk neuroblastoma. Cancer Chemother Pharmacol 74(5):1047-1055. doi:10.1007/s00280-014-2575-9

15. Ozkaynak MF, Gilman AL, Yu AL, London WB, Sondel PM, Smith MA, Seeger R, Reynolds CP, Maris JM, Park JR (2014) A comprehensive safety trial of chimeric antibody 14.18 (ch14.18) with GM-CSF, IL-2, and isotretinoin in high-risk neuroblastoma patients following myeloablative therapy: A Children's Oncology Group study. J Clin Oncol 32(5s):suppl. abstract 10044 